\title{
Assembly Sequence Planning Using Neural Network Approach
}

Cem Sinanoglu and Huseyin Riza Borklu

\section{Introduction}

The competition between manufacturing firm's makes it necessary that those firms must supply highly quality goods with shorter time and chipper for survive in the international market. The intensive research in this field aims at augmenting methods and tools for product development and manufacturing. By the use of new and efficient methods it is possible to shorten the time from design to manufacturing and reduce the mistakes originating from humans. Therefore, full automation in the manufacturing processes can be accomplished.

An assembly can be defined as the overall function of individual parts after joining each other's, each of which has an independent function. It is possible to divide an assembly into various subassemblies depending on its complexity levels (Pahl \& Beitz, 1988). Although intensive research efforts in the field of assembly sequence planning, there are still some problems to be solved. For example, there exist some shortcomings to support for full automatic assembly sequence planning or to obtain assembly plans for large systems (Singh, 1997).

By the development of efficient assembly planning systems it is possible to provide some advantages in both areas:

- CAD automation as well as

- Manufacturing performed by the use of robots

In order to develop a system that addresses computer-aided assembly sequence planning, these issues should be taken into consideration:

- The connectivity structure of parts and/or subassemblies that are used in the assembly system 
- If they have connectivity properties, then what are the theatrical number of their different connection ways

- Finally the choice of the most optimum assembly process among various alternatives

Basic approach for finding the most suitable assembly sequences is to represent assembly system in a space where it is possible to explore all different assembly sequences. Thus, some criterion may be used to obtain these sequences. Then optimum assembly sequence can be selected by the application of some other criterion. This criterion may include: the number of tool changes, part replacement and clamping on the jigs, concurrency in operations, reliable subassemblies, etc (Kandi \& Makino, 1996). A new approach may be stated as a rank evaluation from new scanning factor. But this may not exactly show the differences between operation time and costs.

The initial assembly planning system was inquired to user data necessity for operation and it was formed to assembly sequences with given data. It was worked with the user interaction (De Fazio, 1987, Homem de Mello \& Sanderson, 1991). The later work concentrated on assembly sequence planning systems based on geometric reasoning capability and full automatic (Homem de Mello, 1991). Then, the system included some modules that allow assembly sequence plans to be controlled and to be tested.

Since many assembly sequences share common subsequences, attempts have been made to create more compact representations that can encompass all assembly sequences. Therefore, the works in this field are graph-based approaches to represent all feasible assembly sequences $(F A S)$.

This paper presents a neural-network-based computational scheme to generate feasible assembly sequences for an assembly product. The inputs to the networks are the collection of assembly sequence data. This data is used to train the network using the Back propagation $(B P)$ algorithm. The neural network model outperforms the feasible assembly sequence-planning model in predicting the optimum assembly sequences.

\section{System Definition}

This assembly planning system is used to assembly's connection graph $(A C G)$ for the representation product. Parts and relations among these parts are represented by this graph. Contact relations between parts are supplied by scan- 
ning module. Scanning module scans various assembly views of any product whose assembly view is produced by $C A D$ packet program in the computer environment and determines to contact and interference relations between parts (Sinanoğlu \& Börklü, 2004).

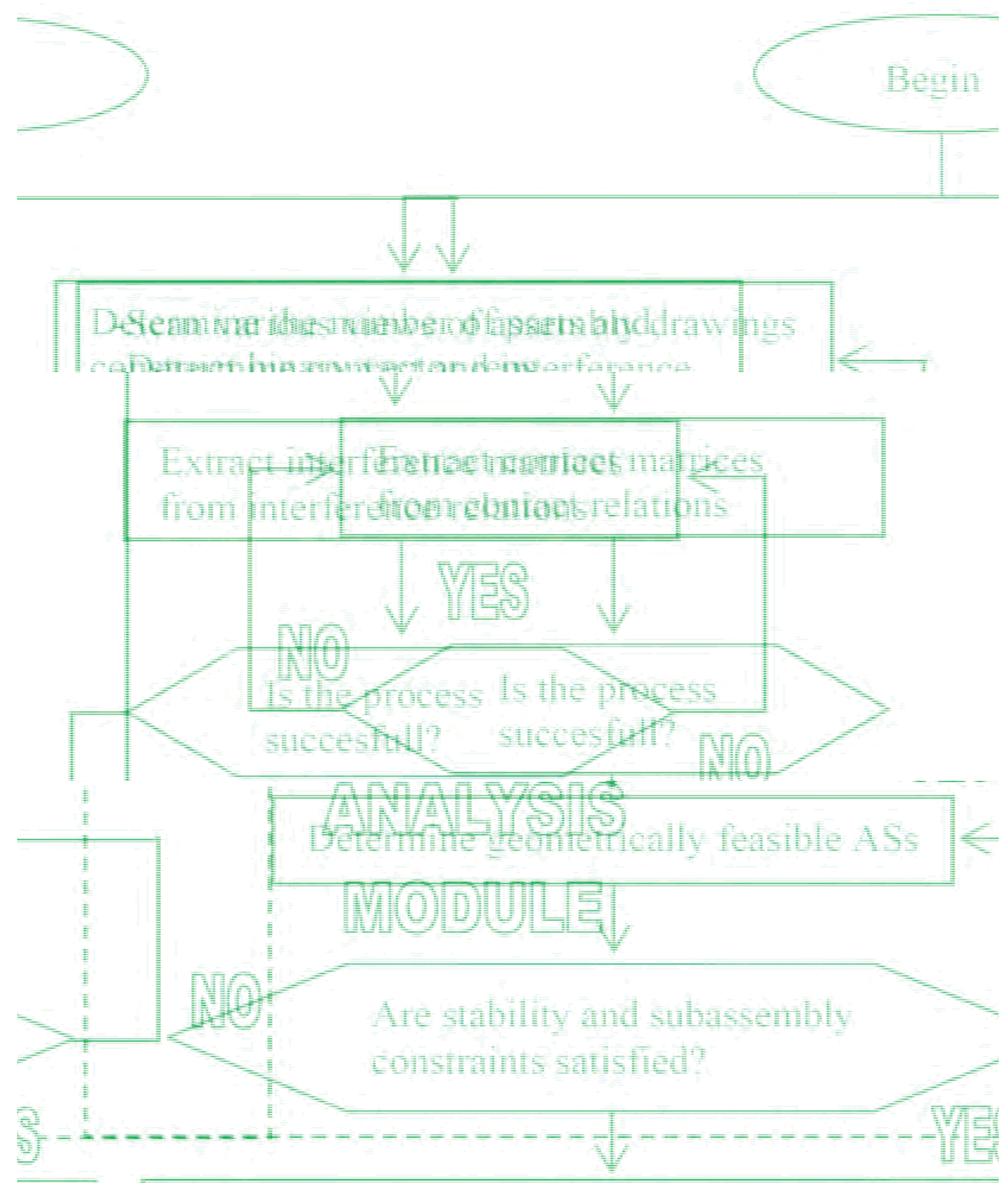

Figure 1. Block diagram of proposed assembly planning system 
These relations are formed as a matrix. The system constitutes $A C G$ of product to be assembled, by applying Boolean operators on the elements of the contact matrices according to certain rules. Moreover, interference relations are also accumulated in a form of interference matrices for determination of geometric feasibility of assembly states later (Sinanoğlu \& Börklü, 2005).

Block diagram of assembly system is shown in Fig. 1. In the assembly planning system, binary vectors represent assembly states. Therefore, all binary vector representations, whether corresponding to assembly states or not, are produced by the system. By evaluating $A C G$ and binary vector representations simultaneously with the scanning module, vector representations corresponding to assembly states are determined.

Some of the assembly states cannot take part in a feasible assembly sequence. The determination of the assembly states not corresponding to feasible assembly sequence is achieved with the analysis module. The analysis module controls all assembly states according to stability, subassembly and geometric feasibility constraints. Boolean operators apply to the elements of interference matrices determination of geometric feasibility. The feasible assembly states and assembly sequences are represented by a directed graph (Homem de Mello \& Arthur, 1990). Assembly states supplying constraints are settled down in the nodes of directed graph hierarchically by the system.

Any path from the root node to terminal node in the directed graph corresponds to feasible assembly sequence. The optimum one is selected from the feasible assembly sequences with optimization module. The neural network approach has been employed for analyzing feasible assembly sequences for sample product. Due to parallel learning structure of the network, the proposed neural network has superior performance to analyze these systems.

\section{Artificial Neural Networks}

An artificial neural network (or simply a neural network-NN) is a biologically inspired computational structure, which consists of processing elements (neurons) and connections between them with coefficients (weights) bound to the connections.

Training and a recall algorithm are associated to every NN. NN are also called connectionist models because of the main role of the connections the weights are the result of the training phase; they are the "memory" of the structure. The connection weights change during learning (training). 
A structure of a typical biological neuron is shown in Fig. 2(a). It has many inputs (in) and one output (out). The connections between neurons are realized in the synapses. An artificial neuron is defined by (Fig. 2(b)):

- Inputs $x_{1}, x_{2}, \ldots \ldots, x_{n}$

- Weights, bound to the inputs $w_{1}, w_{2}, \ldots, w_{n}$

- An input function $(f)$, which calculates the aggregated net input

- Signal $U$ to the neuron (this is usually a summation function)

- An activation (signal) function, which calculates the activation

- Level of the neuron: $O=g(U)$

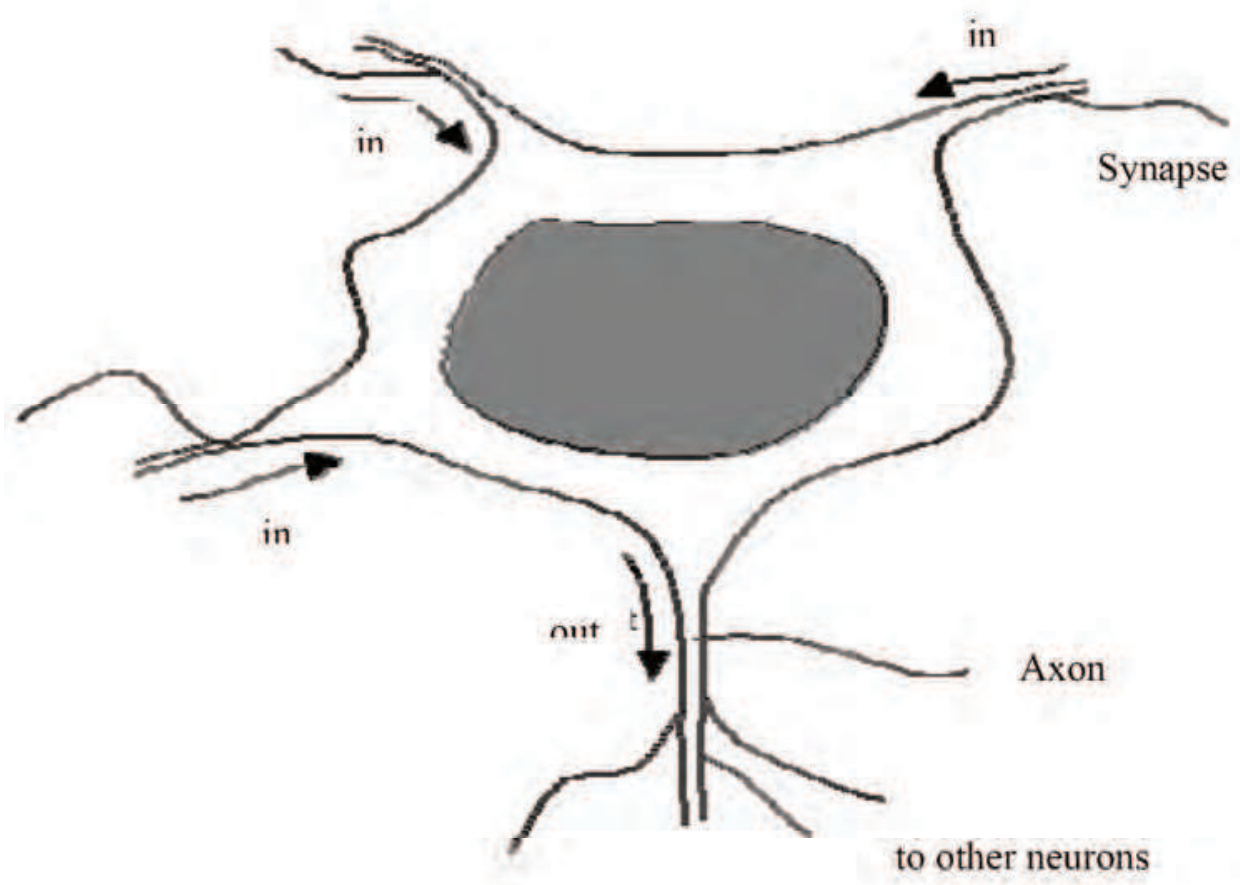

Figure 2(a). Schematic view of a real neuron 


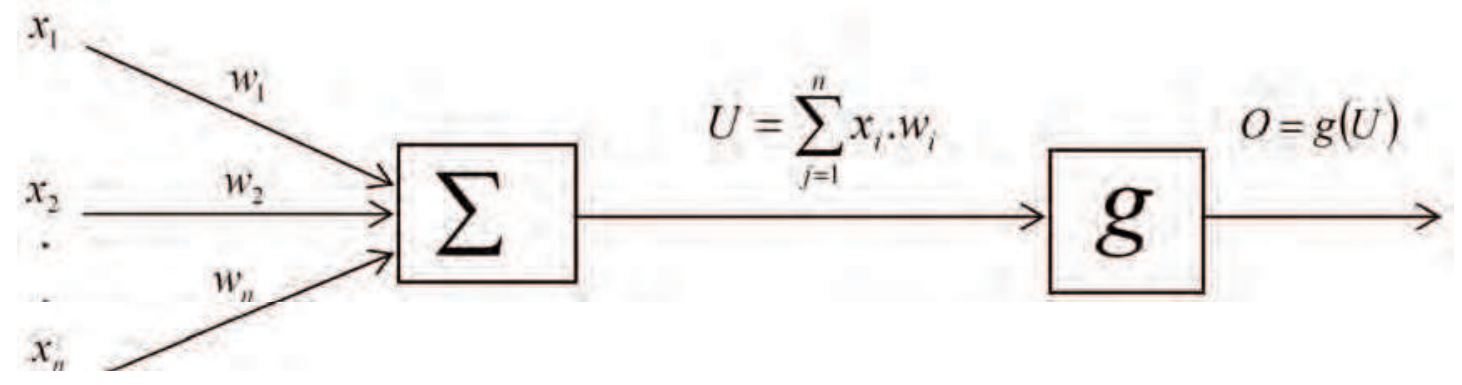

Figure 2(b) Schematic representation of the artificial neural network

Fig. 2(c) shows the currently loaded network. The connections can represent the current weight values for each weight. Squares represent input nodes; circles depict the neurons, the rightmost being the output layer. Triangles represent the bias for each neuron. The neural network consists of three layer, which are input, output and hidden layers. The input and outputs data are used as learning and testing data.

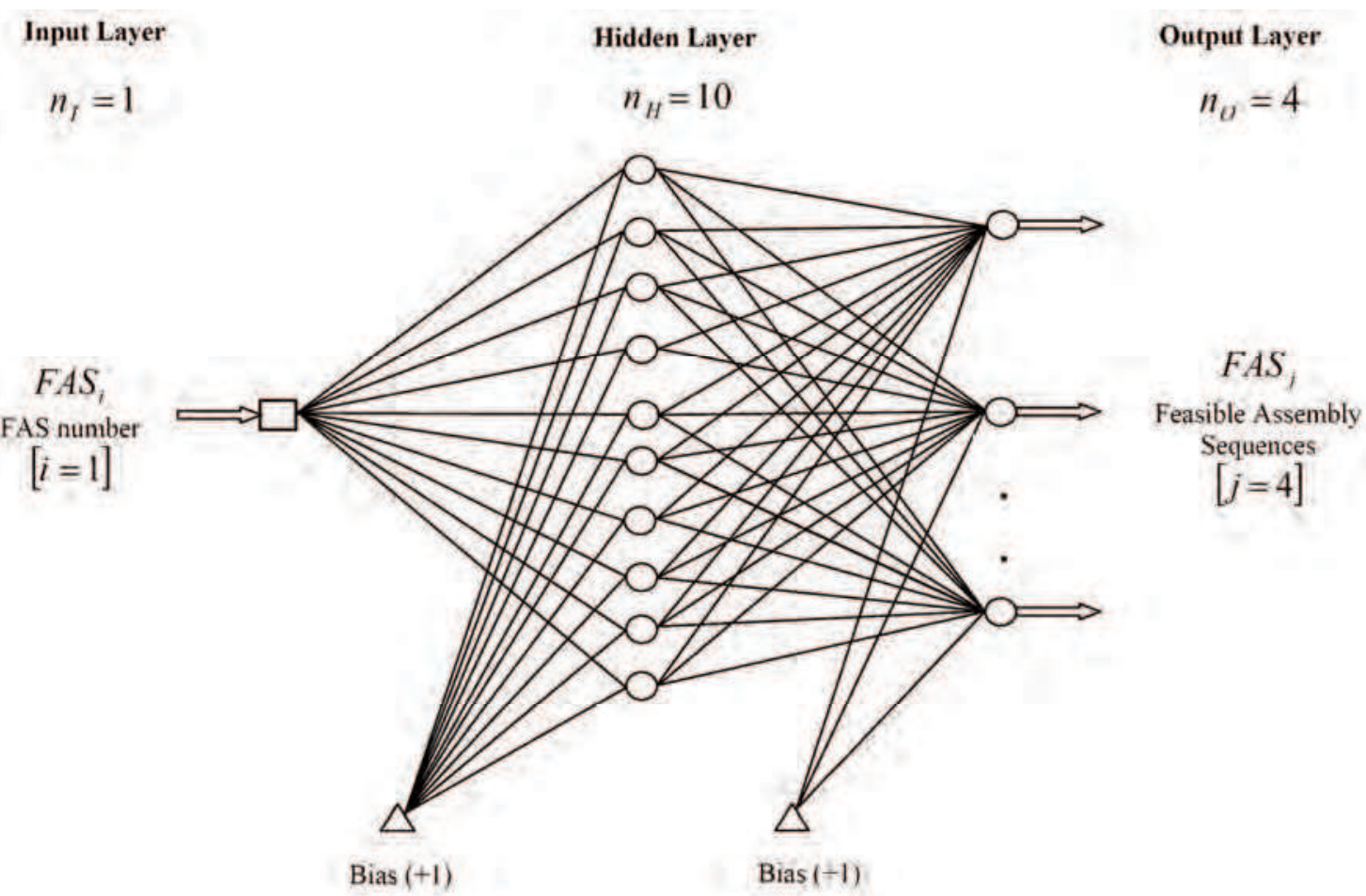

Figure 2(c) Currently loaded network 
The most important and time-consuming part in neural network modeling is the training process. In some cases the choice of training method can have a substantial effect on the speed and accuracy of training. The best choice is dependent on the problem, and usually trial-and-error is needed to determine the best method. In this study, logistic function and back-propagation learning algorithm are employed to train the proposed NN.

Back propagation algorithm is used training algorithm for proposed neural networks. Back propagation is a minimization process that starts from the output and backwardly spreads the errors (Canbulut \& Sinanoğlu, 2004). The weights are updated as follows;

$$
\Delta w_{i j}(t)=-\eta \frac{\partial E(t)}{\partial w_{i j}(t)}+\alpha \Delta w_{i j}(t-1)
$$

where, $\eta$ is the learning rate, and $\alpha$ is the momentum term.

In this study, the logistic function is used to hidden layers and output layers. Linear function is taken for input layer. Logistic function is as follows;

$$
y=f(x)=\frac{1}{1+e^{-x}}
$$

Its derivative is;

$$
\frac{\partial y}{\partial x}=y \cdot(1-x)
$$

The linear function is;

$$
y=f(x)=x
$$

Its derivative is;

$$
\frac{\partial y}{\partial x}=1
$$

Training and structural parameters of the network are given in Table 1. 


\begin{tabular}{|c|c|c|c|c|c|c|c|}
\hline $\begin{array}{c}\text { Proposed } \\
\text { Neural } \\
\text { Network }\end{array}$ & $\eta$ & $\mu$ & $n_{I}$ & $n_{H}$ & $n_{O}$ & $N$ & AF \\
\cline { 2 - 8 } & 0.1 & 0 & 1 & 10 & 4 & 500000 & $\log$ istic \\
\hline
\end{tabular}

Table 1. Training and structural parameters of the proposed network

\section{Modeling of Assembly System}

An assembly is a composition of interconnected parts forming a stable unit. In order to modelling assembly system, it is used $A C G$ whose nodes represent assembling parts and edges represent connections among parts. The assembly process consists of a succession of tasks, each of which consists of joining subassemblies to form a larger subassembly. The process starts with all parts separated and ends with all parts properly joined to form the whole assembly. For the current analyses, it is assumed that exactly two subassemblies are joined at each assembly task, and that after parts have been put together, the remain together until the end of the assembly process.

Due to this assumption, an assembly can be represented by a simple undirected graph $\langle P, C\rangle$, in which $P=\left\{p_{1}, p_{2}, \ldots, p_{N}\right\}$ is the set of nodes, and $C=\left\{c_{1}, c_{2}, \ldots, c_{L}\right\}$ is the set of edges. Each node in $P$ corresponds to a part in the assembly, and there is one edge in $C$ connecting every pair of nodes whose corresponding parts have at least one surface contact.

In order to explain the modeling of assembly system approach better way used for this research, we will take a sample assembly shown as exploded view in Fig. 3. The sample assembly is a pincer consisting of four components that are: bolt, left-handle, right-handle and nut. These parts are represented respectively by the symbols of $\{a\},\{b\},\{c\}$ and $\{d\}$. For this particular situation, the connection graph of assembly has the set of the nodes as $P=\{a, b, c, d\}$ and the set of the connections as $C=\left\{c_{1}, c_{2}, c_{4}, c_{5}\right\}$.

The connections or edges defining relationships between parts or nodes can be stated as: $c_{1}$ between parts $\{a\}$ and $\{b\}, c_{2}$ between parts $\{a\}$ and $\{d\}, c_{3}$ between parts $\{c\}$ and $\{d\}, c_{4}$ between parts $\{a\}$ and $\{c\}$ and finally $c_{5}$ between parts $\{b\}$ and $\{c\}$. 


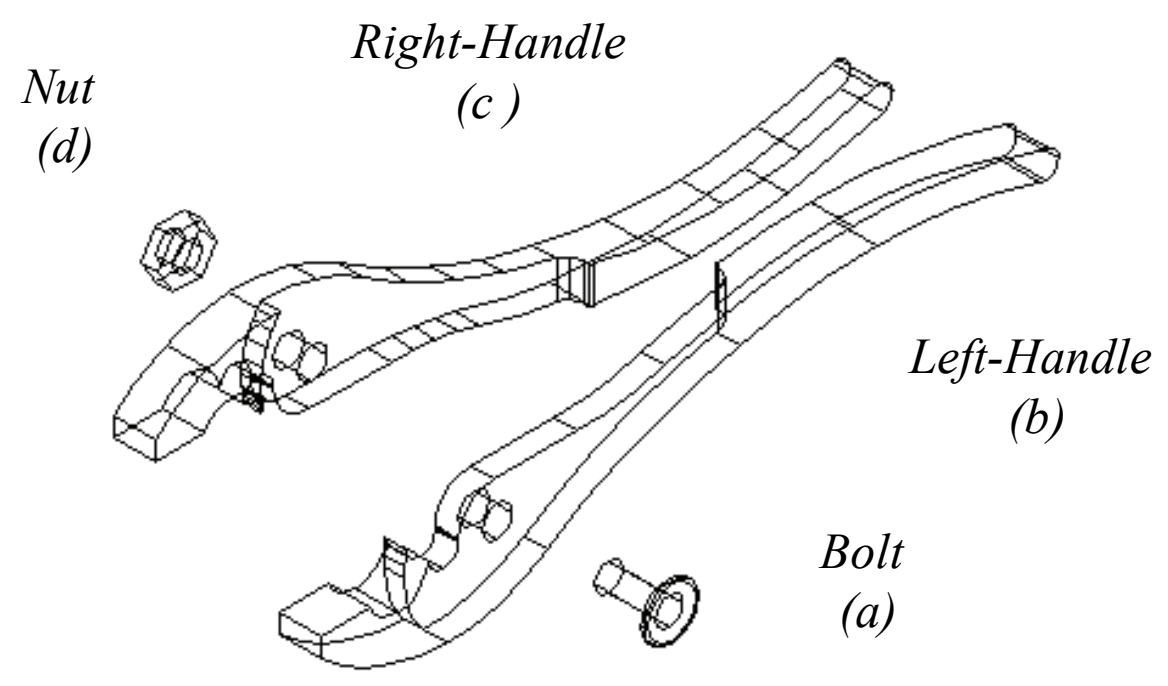

Figure 3. The pincer assembly system

\subsection{Definition of Contact Matrices and $A C G$}

The contact matrices are used to determine whether there are contacts between parts in the assembly state. These matrices are represented by a contact condition between a pair of parts as an $\{A, B\}$. The elements of these matrices consist of Boolean values of true (1) or false (0). For the construction of contact matrices, the first part is taken as a reference. Then it is examined that whether this part has a contact relation in any $i$ axis directions with other parts. If there is, that relation is defined as true (1), else that is defined as false (0).

The row and column element values of contact matrices in the definition of six main coordinate axis directions are relations between parts and that constitutes a pincer assembly. To determine these relations, the assembly's parts are located to rows and columns of the contact matrices. Contact matrices are square matrices and their dimensions are $4 \times 4$ for pincer.

For example, $[a, b]$ element of $B$ contact matrix in $i$ direction is defined to whether there exists any contacts or not between parts $\{a\}$ and $\{b\}$ for the related direction and the corresponding matrix element may have the values of (1) and (0), respectively. 

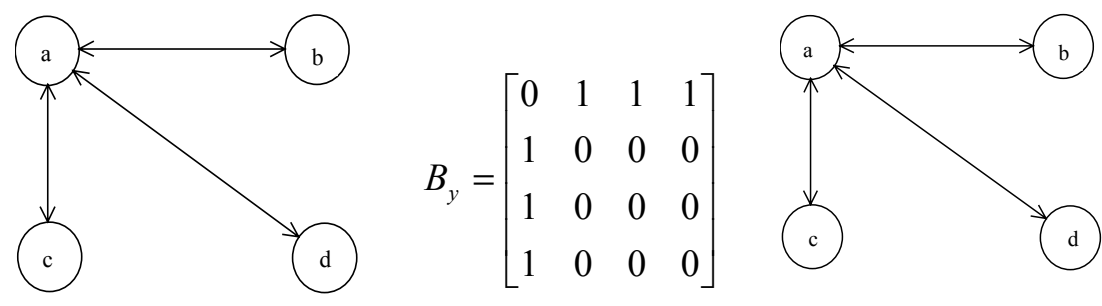

$B_{z}=\left[\begin{array}{llll}0 & 1 & 1 & 1 \\ 1 & 0 & 0 & 0 \\ 1 & 0 & 0 & 0 \\ 1 & 0 & 0 & 0\end{array}\right]$
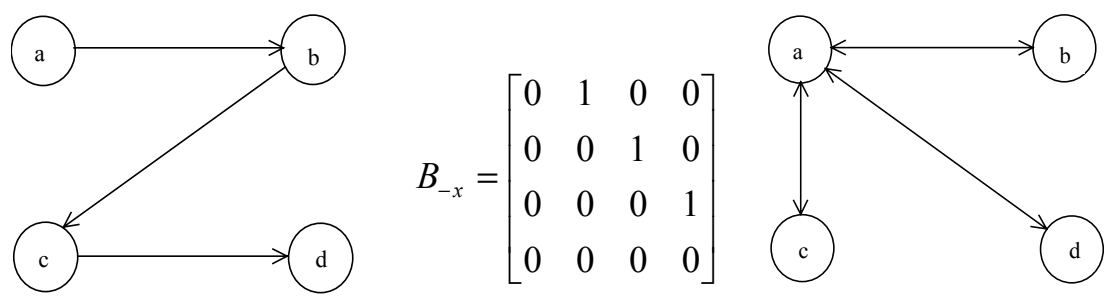

$B_{-y}=\left[\begin{array}{llll}0 & 1 & 1 & 1 \\ 1 & 0 & 0 & 0 \\ 1 & 0 & 0 & 0 \\ 1 & 0 & 0 & 0\end{array}\right]$

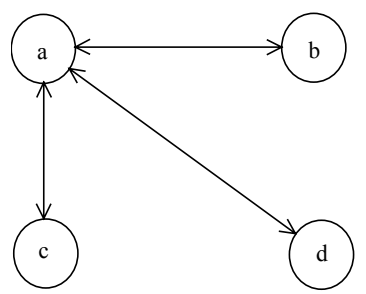

$$
B_{-z}=\left[\begin{array}{llll}
0 & 1 & 1 & 1 \\
1 & 0 & 0 & 0 \\
1 & 0 & 0 & 0 \\
1 & 0 & 0 & 0
\end{array}\right]
$$

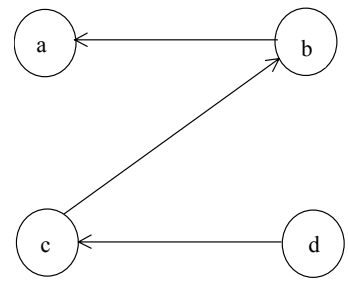

$B_{x}=\left[\begin{array}{llll}0 & 0 & 0 & 0 \\ 1 & 0 & 0 & 0 \\ 0 & 1 & 0 & 0 \\ 0 & 0 & 1 & 0\end{array}\right]$

Figure 4. Contact matrices and their graph representations

In this system, in order to get contact matrices in the direction of Cartesian coordinate axis, assembly view of pincer system was used. These matrices were automatically constructed (Sinanoğlu \& Börklü, 2004). Contact matrices of the pincer assembly system are also shown in Fig. 4.

The connection graph can be obtained from contact matrices. To construct $A C G$, contact conditions are examined in both part's sequenced directions. For instance, in the manner of $\{a, b\}$ sequenced pair of parts, it is sufficient to determine contacts related sequenced direction so that its contact in any direction. Due to this reason, an $[\vee: O r]$ operator is applied to these parts. But it is 
also necessary contacts in any direction for inverse sequenced pairs of parts in the $A C G$. If these values are (1) for every sequenced pair of parts, then there should be edges between corresponding nodes of the $A C G$. For this purpose, every pair of parts must be determined.

- $\{a, b\},\{b, a\}$ Sequenced pair of parts

To investigate whether there is an edge between $\{a\}$ and $\{b\}$ in $A C G$ or not, it should be searched contact relations for these pairs of parts. Table 2 shows contact relations regarding $\{a, b\}$ and $\{b, a\}$ pairs of parts.

\begin{tabular}{|c|c|c|c|c|c|c|c|c|}
\hline$c_{1} \Rightarrow(a \div b)$ & $x$ & $y$ & $z$ & $-x$ & $-y$ & $-z$ & $\vee:$ Or $\Rightarrow$ & $\wedge:$ And $\Downarrow$ \\
\hline$a / b$ & 0 & 1 & 1 & 1 & 1 & 1 & 1 & 1 \\
\hline$b / a$ & 1 & 1 & 1 & 0 & 1 & 1 & 1 & 1 \\
\hline
\end{tabular}

Table 2. Contact relations of $\{a, b\}$ and $\{b, a\}$ pairs of parts

In this table, $\{a, b\}$ sequenced pair of parts is supplied to at least one contact condition in the related direction of $(0 \vee 1 \vee 1 \vee 1 \vee 1 \vee 1=1) .\{b, a\}$ pair of parts is also supplied to at least one contact in the related direction of $(1 \vee 1 \vee 1 \vee 0 \vee 1 \vee 1=1)$. An $(\wedge:$ And $)$ operator is applied to these obtaining values. Because, these parts have at least one contact in each part sequenced direction, there is an edge between parts in the $A C G$. This connection states an edge in the $A C G$ shown in Fig. 5.

If similar method is applied to other pairs of parts: $\{a, d\},\{d, a\},\{b, c\},\{c, b\}$, $\{c, d\},\{d, c\},\{a, c\}$ and $\{c, a\}$, the results should be (1). Therefore, there are edges between these pairs in $A C G$.

The graph representation of this situation is shown in Fig. 5, where there is no edge between parts $\{b\}$ and $\{d\}$. Therefore, these parts do not have any contact relations.

Fig. 5 shows the pincer graph of connections. It has four nodes and five edges (connections). There is no contact between the left-handle and the nut. There- 
fore, the graph of connections does not include an edge connecting the nodes corresponding to the left-handle and the nut. By the use of the contact matrices and applying some logical operators to their elements, it is proved that it is supplied to one connection between two part in $A C G$ not all contacts between them are established in every direction.

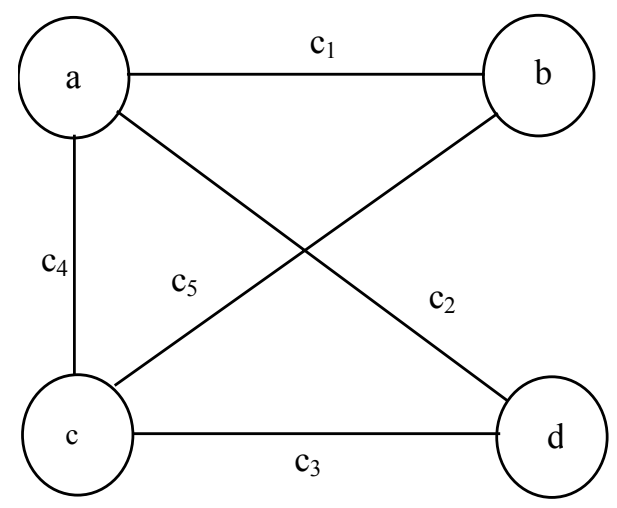

Figure 5. The graph of connections for four-part pincer assembly

\section{Determination of Binary Vector Representation and Assembly States $(A S s)$}

The state of the assembly process is the configuration of the parts at the beginning (or at the end) of an assembly task. The configuration of parts is given by the contacts that have been established. Therefore, in the developed approach an L-dimensional binary vector can represent a state of the process $\left(x=\left\{x_{1}, x_{2}, \ldots, x_{L}\right\}\right)$. Elements of these vectors define the connection data between components. Based upon the establishment of the connections, the elements of these vectors may have the values of either (1) or (0) at any particular state of assembly task. For example, the $i^{\text {th }}$ component $x_{i}$ would have a value of true (1) if the $i^{\text {th }}$ connection were established at that state. Otherwise, it would have a value of false (0). Moreover, every binary vector representations are not corresponding to an assembly state. In order to determine assembly states, the established connections in binary vectors and $A C G$ are utilised together. 
There are five edges in the example $A C G$. Because of that, the elements of vectors are five and the 5-dimensional binary vector of can represent that $\left[c_{1}, c_{2}, c_{3}, c_{4}, c_{5}\right]$. For instance, the initial state of the assembly process for the product shown in Fig. 3 can be represented by binary vector [FFFFF] whereas the final state can be represented by [TTTTT].

If the first task of the assembly process is the joining of the bolt to nut, the second state of the assembly process can be represented by [FTFFF].

For example, an assembly sequence for pincer system can be represented as follows:

$$
([F F F F F],[F T F F F],[T T T F F],[T T T T T]) \quad([00000],[01000],[11100],[11111])
$$

The first element of this list represents the initial state of the assembly process. The second element of the list shows the second connection $c_{2}$ between bolt and nut. The third element represents $c_{1}$ connection between right-handle and bolt and $c_{3}$ connection between right-handle and nut. The last element of the list is [11111] and it means that every connection has been established.

In the developed planning system, first of all binary vector representations must be produced. The purpose of that it is classified to binary vectors according to the number of established connections. Table 3 shows vector representations for pincer assembly in Fig. 3. There are thirty-two different binary vectors. While some of them correspond to assembly state, some of them are not.

To form assembly sequences of pincer system, vector representations corresponds to assembly states must be determined. In order to determine whether the vector is a state or not, it must be taken into consideration established connections in vector representation. And then it is required that establishing connections must be determined to established connections by $A C G$.

For instance, if the first task of the assembly process is the joining of the bolt to the left-handle, the second state of the assembly process can be represented by [10000]. It is seen in Fig. 6 that it does not necessary to establish any connection so that $c_{1}$ connection between part $\{a\}$ and $\{b\}$ is establish. Therefore, [10000] vector is an assembly state. Therefore, vectors only one established connection form assembly state. 


\begin{tabular}{|c|c|c|c|c|c|}
\hline $\begin{array}{c}\text { LEVEL } \\
0\end{array}$ & $\begin{array}{c}\text { LEVEL } \\
1\end{array}$ & \multicolumn{3}{|c|}{ LEVEL } & $\begin{array}{c}\text { LEVEL } \\
3\end{array}$ \\
\hline \multirow{30}{*}{00000} & \multirow{6}{*}{10000} & & 11100 & & \\
\hline & & 11000 & 11010 & 11110 & \\
\hline & & 10100 & 11001 & 11101 & \\
\hline & & 10010 & 10110 & 11011 & \\
\hline & & 10001 & 10101 & 10111 & \\
\hline & & & 10011 & & \\
\hline & & & 11100 & & \\
\hline & & 11000 & 11010 & 11110 & \\
\hline & & 01100 & 11001 & 11101 & \\
\hline & 01000 & 01010 & 01110 & 11011 & \\
\hline & & 01001 & 01101 & 01111 & \\
\hline & & & 01011 & & \\
\hline & & & 11100 & & \\
\hline & & 10100 & 10110 & 11110 & \\
\hline & & 01100 & 10101 & 11101 & \\
\hline & 00100 & 00110 & 01110 & 10111 & 11111 \\
\hline & & 00101 & 01101 & 01111 & \\
\hline & & & 00111 & & \\
\hline & & & 11010 & & \\
\hline & & 10010 & 10110 & 11110 & \\
\hline & 00010 & 01010 & 10011 & 11011 & \\
\hline & & 00110 & 01110 & 10111 & \\
\hline & & 00011 & 01011 & 01111 & \\
\hline & & & 00111 & & \\
\hline & & & 11001 & & \\
\hline & & 10001 & 10101 & 11101 & \\
\hline & & 01001 & 10011 & 11011 & \\
\hline & 00001 & 00101 & 01101 & 10111 & \\
\hline & & 00011 & 01011 & 01111 & \\
\hline & & & 00111 & & \\
\hline
\end{tabular}

Table 3. Hierarchical levels of binary vector representations for pincer assembly system 
Moreover, some of vectors do not correspond to an assembly state. For instance, in the [10001] vector, connections of $c_{1}$ between $\{a\}$ and $\{b\}, c_{5}$ between $\{b\}$ and $\{c\}$ have been established (1). It has been necessary to establish $c_{4}$ connection between $\{a\}$ and $\{c\}$ so that these connections have been established (Fig. 6). But this connection has not been established in [10001], [10001] vector is not an assembly state.

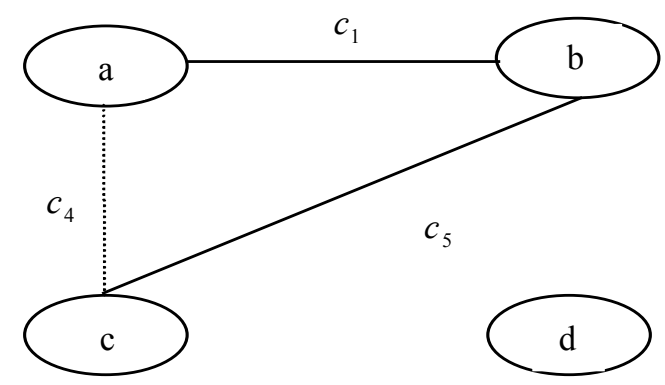

Figure $6 c_{1}, c_{5}$ and $c_{4}$ connections in [10001] vector

There are thirteen assembly states in pincer assembly system. These are; [00000],[10000],[01000],[00100],[00010],[00001],[11000],[10100],[01001],[00101],[10011],[01110],,[11111]

\section{Productions and Representation of Assembly Sequences}

Given an assembly whose graph of connections is $\langle P, C\rangle$, a directed graph can be used to represent the set of all assembly sequences (Homem de Mello \& Lee, 1991). The directed graph of feasible assembly sequences of an assembly whose set of parts is $P$ is the directed graph $\left\langle x_{p}, T_{p}\right\rangle$ in which, $x_{p}$ is the assembly's set of stable states, and $T_{p}$ is the assembly's set of feasible state transitions.

In the pincer assembly, $P=\{a, b, c, d\}$ is the assembly's set of parts or set of nodes, $C=\left\{c_{1}, c_{2}, c_{3}, c_{4}, c_{5}\right\}$ is the assembly's set of connections or set of edges. $\left\langle x_{p}, T_{p}\right\rangle$ corresponds to directed graph of pincer system. A path in the directed graph of feasible assembly sequences $\left\langle x_{p}, T_{p}\right\rangle$ whose initial node is $\Theta_{I}=\{\{a\},\{b\},\{c\},\{d\}\}$ and whose terminal node are $\Theta_{F}=\{\{a, b, c, d\}\}$. Vector representations of these sets are [00000] and [11111] respectively. 
Assembly states not correspond to feasible assembly sequences must eliminate by some assembly constraints. In this study, three assembly constraints are applied to assembly states. These are subassembly, stability and geometric feasibility constraints.

The subassembly constraint defines feasibility of subassembly of set of partitions to established connections in assembly states. In order to form a subassembly of a set of partition, it is not a set of partition contains a pair of part has not contact relation in the $A C G$. Therefore, in pincer assembly bolt and left-handle has not contact relations. Because of that it is not supplied to subassembly constraint set partitions contains $\{b, d\}$ set of partition.

The second constraints is stability. A subassembly is said to be stable if its parts maintain their relative position and do not break contact spontaneously. All one-part subassemblies are stable.

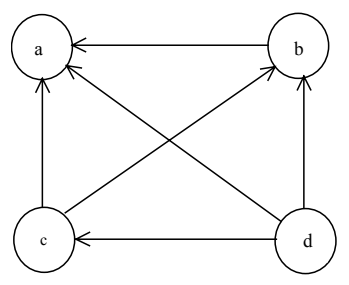

$$
A_{x}=\left[\begin{array}{llll}
1 & 1 & 1 & 1 \\
0 & 1 & 1 & 1 \\
0 & 0 & 1 & 1 \\
0 & 0 & 0 & 1
\end{array}\right]
$$

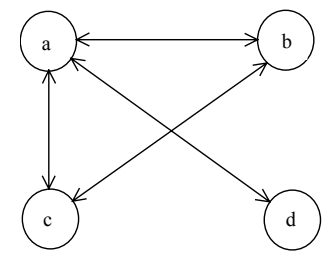

$$
A_{y}=\left[\begin{array}{llll}
1 & 0 & 0 & 0 \\
0 & 1 & 0 & 1 \\
0 & 0 & 1 & 1 \\
0 & 1 & 1 & 1
\end{array}\right]
$$
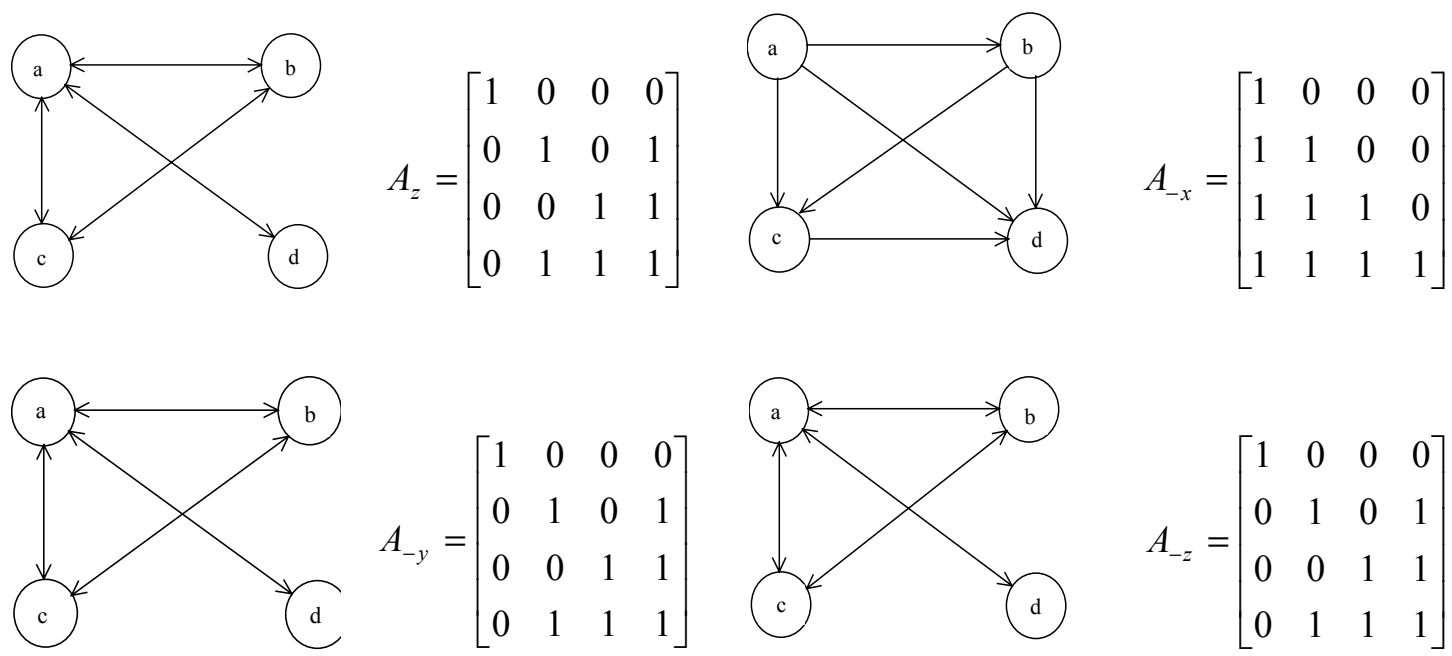

$$
A_{-z}=\left[\begin{array}{llll}
1 & 0 & 0 & 0 \\
0 & 1 & 0 & 1 \\
0 & 0 & 1 & 1 \\
0 & 1 & 1 & 1
\end{array}\right]
$$

Figure 7. Interference matrices and their graph representations for pincer assembly 
The last constraint is geometric feasibility. An assembly task is said to be geometrically feasible if there is a collision-free path to bring the two subassemblies into contact from a situation in which they are far apart.

Geometric feasibility of binary vectors correspond to assembly states are determined by interference matrices. The elements of interference matrices were taken into consideration interference conditions during the joining parts. In the determination of geometric feasibility, it is applied to elements of interference matrices $(\wedge)$ and $(\vee)$ logical operators. At this operation, it must be utilise established connections and that is joining pairs of part. In order to, whether binary vector representations corresponds to assembly states are geometrically feasible or not, it is necessary to applying Cartesian product between sequenced pairs of parts which are representing established connections and parts which are not in this sequenced pairs.

In the determination of interference matrices elements, it is taken into consideration interference while the reference part is moving with another part along with related axis direction. If it is interference during this transformation motion, interference matrices elements are (0) if not are defined as (1).

For instance, in the $A_{x}$ matrice the movement of part bolt is interfered to movement along with $\{+x\}$ axis by other parts. Therefore, the first row elements of $A_{x}$ matrice defined to interference among parts along this axis are (1). But the movement of left-handle along with $\{+x\}$ axis does not interfere any parts (Fig. 3). This interference relation is illustrated to designate (0) value by element of second row and third column in $A_{x}$ matrice. These matrices are also formed automatically from various assembly views.

Graph representations for the pincer assembly and construction of their interference matrices can be also determined as follows (Fig. 7).

In order to determine whether assembly states are geometrically feasible or not, it is necessary to apply Cartesian product between sequenced pairs of parts which represent established connections and parts which are not in this sequenced pairs of parts. In this situation, different interference tables are obtained and these tables are used to check geometric feasibility.

- $[01000]$ Assembly State

In this assembly state, connection of $c_{2}$ between part $\{a\}$ and $\{d\}$ has been established. To determine geometric feasibility of this assembly state, parts 
without in established conditions are taken. Those are $\{b\}$ and $\{c\} .\{a, d\}$ sequenced pair of part represents established connection $c_{2}$. Cartesian product, which is between $\{a, d\}$ and $\{b\}$ is given as follows.

$(a, d)(b) \Rightarrow(a, b)(d, b)$

Table 4 shows interference's of $\{a, b\}$ sequenced pair of part.

\begin{tabular}{|c|c|c|c|c|c|c|c|}
\hline$c_{2} \Rightarrow(a \div d)$ & $x$ & $y$ & $z$ & $-x$ & $-y$ & $-z$ & \\
\hline$a / b$ & 1 & 0 & 0 & 0 & 0 & 0 & $\vee \Rightarrow$ \\
\cline { 1 - 5 } &
\end{tabular}

Table 4. Interference of $\{a, b\}$ sequenced pair of part

Another part without parts constituted assembly state is part $\{c\}$. As a result of Cartesian product is $(a, d)(c) \Rightarrow(a, c)(d, c)$. Table 5 shows interference of them.

\begin{tabular}{|c|c|c|c|c|c|c|c|}
\hline$c_{2} \Rightarrow(a \div d)$ & $x$ & $y$ & $z$ & $-x$ & $-y$ & $-z$ & \\
\hline$a / c$ & 1 & 0 & 0 & 0 & 0 & 0 & \\
\hline$d / c$ & 0 & 1 & 1 & 1 & 1 & 1 & \\
\hline$(a / c \wedge d / c) \Downarrow$ & 0 & 0 & 0 & 0 & 0 & 0 & $\vee \Rightarrow$ \\
\hline & & & & & & & 0 \\
\hline
\end{tabular}

Table 5. Interference relations of $\{a, c\}$ and $\{d, c\}$ pairs

Although it is geometrically feasible (1) to disassemble from $\{a, d\}$ to $\{b\}$, it is not geometrically feasible $(0)$ to disassemble from $\{a, d\}$ to $\{c\}$. As a result of $(\wedge)$ logical operator is $(0)(1 \wedge 0=0)$. This result explained that [01000] assembly state is geometrically unfeasible.

Moreover, other assembly states of [00100] and [00001] are geometrically feasible, but [00010] is geometrically unfeasible. Similarly, [11000],[10100] and [00101] assembly states contain two established connection are geometrically feasible, but [01001] is not geometrically feasible. Moreover, [10011] assembly state contains three connections that are geometrically feasible but [01110] is geometrically unfeasible. [11111] vector is also geometrically feasible. The number of nodes is reduced from 15 to 8 in the di- 
rected graph by applying assembly constraints. The assembly states supplied to these constraints are as follows:

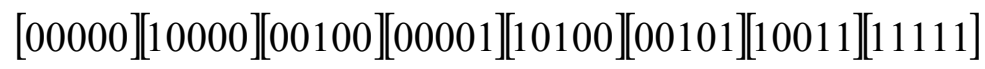

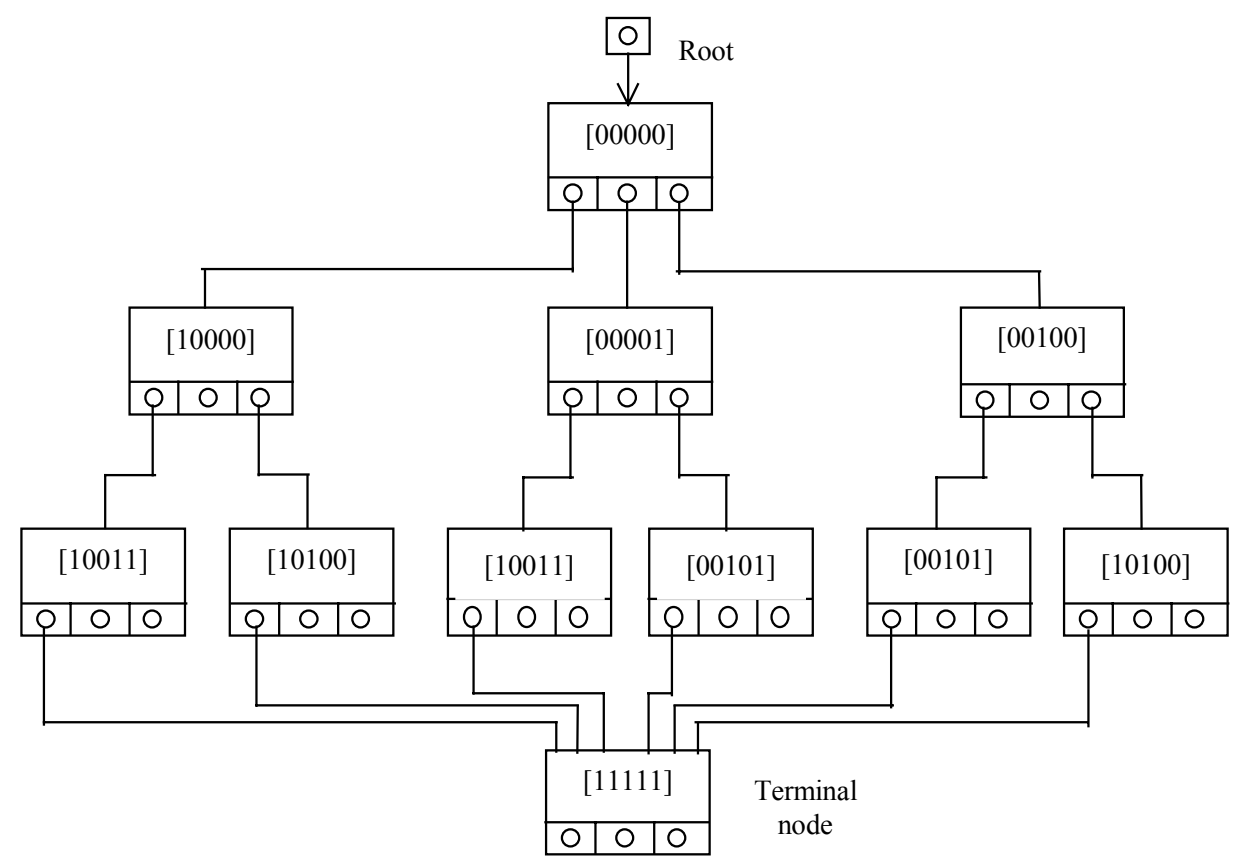

Figure 8. Constrained directed graph for pincer system

Fig. 8 shows the directed graph of feasible assembly sequences after applied constraints. A path in the directed graph of feasible assembly sequences whose initial node is [00000] and terminal node is [11111] corresponds to a feasible assembly sequences for pincer. The feasible assembly sequences for pincer assemblies are as follows:

$\begin{array}{ll}F A S-I & {[00000][10000][10011][11111]} \\ F A S-I I & {[00000][10000][10100][11111]} \\ F A S-I I I & {[00000][00001][10011][11111]} \\ F A S-I V & {[00000][00001][00101][11111]} \\ F A S-V & {[00000][00100][00101][11111} \\ F A S-V I & {[00000][00100][10100][1111]}\end{array}$


For example, in the third assembly sequence for pincer system, at first, the left handle is joined to right handle with connection of $c_{5}$. After that this subassembly is joined by using the bolt with the connections of $c_{1}$ and $c_{4}$. Finally, the nut fixes all parts.

\section{Optimization of Assembly Sequences}

Developed assembly planning system can be determined to find the optimum assembly sequence. In this section, an optimization approach is explained. For this purpose, the pincer assembly system is taken as an example. It has been obtained from feasible assembly sequences in previous sections. In order to optimize the assembly sequence, two criteria are developed, weight and the subassembly's degree of freedom. First certain costs are assigned to edges of directed graph depend on these criteria, and then the total cost of each path from root node to terminal is calculated the minimum cost sequence is selected as an optimum one.

\subsection{Optimization of Weight Criterion}

In order to determine the optimum assembly sequence, all assembly states in an assembly sequence must be taken into consideration. The heaviest and bulkiest part is selected as a base part and then the assembly sequence continues from heavy to light parts. The parts with the least volume, i.e. connective parts, like bolts and nuts must be assembled last (Bunday, 1984). The weights and volumes of parts were calculated automatically with a $C A D$ program.

Therefore, determination of the costs of assembly states is necessary to obtain an optimum feasible assembly sequence. After that these costs are used as a reference to different assembly states. Calculated weight costs of assembly states in the assembly sequence are compared with reference weights. The difference of weight is multiplied by unit weight value (100). The weights of parts of the pincer system are as follows: Bolt $(0.0163 \mathrm{~kg})$, left-handle $(0.3843 \mathrm{~kg})$, right-handle $(0.3843 \mathrm{~kg})$ and nut $(0.0092 \mathrm{~kg})$. Using the weight criterion, the total established connection weights of each assembly state in optimum assembly sequence could be determined.

The total weight of assembly states in the optimum sequence according to weight criterion can be defined as; 


$$
O w_{m}=\sum_{i=1}^{n}(W)
$$

where $W$ is the weight of assembly states, $(n)$ is the number of the established connections and $(m)$ is the order of assembly states. $O w_{m}$ is the required weight of assembly states in the optimum assembly sequence.

In order to determine the optimum assembly sequence, the weights of all assembly states in assembly sequences are calculated. This weight is expressed as;

$$
C w_{m}=\sum_{i=1}^{n}(W)
$$

where $W$ is the weight of assembly states, $(n)$ is the number of the established connections and $(m)$ is the sequenced of assembly states.

After that, $\left(O w_{m}\right)$ is used as a reference for different assembly states. Calculated weights of assembly states in assembly sequences are compared with reference weights. This weight difference $(D w)$ is multiplied by unit weight value $(U w v=100)$. The result is the weight costs of any assembly states $(W c)$.

$$
D w_{m}=O w_{m}-C w_{m}
$$

$$
\begin{aligned}
& W c=\sum_{i=1}^{l}\left((W c)_{m}\right)= \\
& \sum_{i=1}^{l}\left(\left(O w_{m}-C w_{m}\right) x U w v\right)=\sum_{i=1}^{l}\left(\left(\left(\sum_{i=1}^{n}(W)\right)_{O}-\left(\sum_{i=1}^{n}(W)\right)_{H}\right) x U w v\right)
\end{aligned}
$$

For example, in the second assembly state of the first feasible assembly sequence ([10000]), $\quad c_{1}$ connection between $\{a\}$ and $\{b\}$ is established. The number of the established connection is $n=1$. The required weight of this state is $C w_{2}=\sum_{i=1}^{1}(W)_{H}=W\left(c_{1}\right)=0.4006 \mathrm{~kg}$.

In the second assembly state, the necessary weight is $O w_{2}=0.7686 \mathrm{~kg}$. 
The difference of weight $D w$ is $D w_{2}=O w_{2}-C w_{2}=0.7686-0.4006=0.368 \mathrm{~kg}$. If $D w$ is multiplied by the unit weight value $(U w v=100)$, the weight cost of $([10000])$ will be calculated as follows; $W c_{2}=D w_{2} \cdot U w v=0.368 \times 100 \cong 37$

The total weight cost of any feasible assembly sequence is expressed as;

$$
W c t=\sum_{i=1}^{z} W c
$$

where $z$ is the total assembly state number of any feasible assembly sequence.

\subsection{Optimization of Subassembly Degree of Freedom Criterion}

The subassembly degree of freedom criterion is based on the selection of parts with low degrees of freedom. So degree of freedom between the subassembly parts is low, the assembly of these parts can be done more easily. It is a unit cost (unit degree of freedom value, Udofv) also used for this criterion. It is "25" and this criterion is more important than the other. Therefore, it is selected as the lower unit cost according to weight criterion, and so that total cost of assembly sequences can be reduced.

It determines degree of interference for pairs of parts connections established along the six main directions of the Cartesian coordinate system. The total degree of freedom (Tdof) for pairs of parts is the product's unit cost.

$$
D O F c=T d o f \times U d o f v
$$

Therefore, in the directed graph costs of degree of freedom according to this criterion are calculated as the degree of freedom for each path from initial node to terminal node. As a result, the minimum cost of the assembly sequence can be selected as an optimum with respect to the degree of freedom criterion. The total weight cost of any feasible assembly sequence is expressed as;

$$
D O F c t=\sum_{i=1}^{z} D O F c
$$


where $z$ is the total assembly state number of any feasible assembly sequence.

The total cost of feasible assembly sequence for any product is expressed as a cost function $f_{c}$;

$$
\begin{aligned}
W c & =\left[\left(O w_{1}+O w_{2}+\ldots . .+O w_{k}\right)-\left(C w_{1}+C w_{2}+\ldots .+C w_{k}\right)\right] x U w v \\
& =\left[\left(\sum_{i=1}^{l} O w_{k}\right)-\left(\sum_{i=1}^{l} C w_{k}\right)\right] x U w v
\end{aligned}
$$

$$
\begin{gathered}
D O F c=T d o f x U d o f v \\
f_{c}=W c t+D O F c t
\end{gathered}
$$

$(l)$ is the number of assembly states in the feasible assembly sequence for any product.

For example, in the second assembly state of the first feasible assembly sequence $([10000]), \quad c_{1}$ connection between $\{a\}$ and $\{b\}$ is established. The number of the established connection is $n=1$. The degree of freedom of this pair of parts is shown in Table 6.

\begin{tabular}{|c|c|c|c|c|c|c|}
\hline$c_{1} \Rightarrow(a \div b)$ & $x$ & $y$ & $z$ & $-x$ & $-y$ & $-z$ \\
\hline$a / b$ & 1 & 0 & 0 & 0 & 0 & 0 \\
\hline$b / a$ & 0 & 0 & 0 & 1 & 0 & 0 \\
\hline
\end{tabular}

Table 6. Degree of freedom between parts $\{a\}$ and $\{b\}$

The total degree of freedom for $([10000])$ is $T d o f=2$. If this value is multiplied by the $U d o f v=25$ unit freedom cost, the result will be "50". Therefore, the degree of freedom cost DOFc for [100000000] assembly state is "50".

Fig. 9 shows feasible assembly sequences and costs of them for the pincer assembly system. The first and third assembly sequences for the pincer system according to the subassembly degree of freedom criterion have been selected 
with an optimum total cost of "300". The weight costs are in parentheses ( ) and the degree of freedom costs are in quotation marks " ".

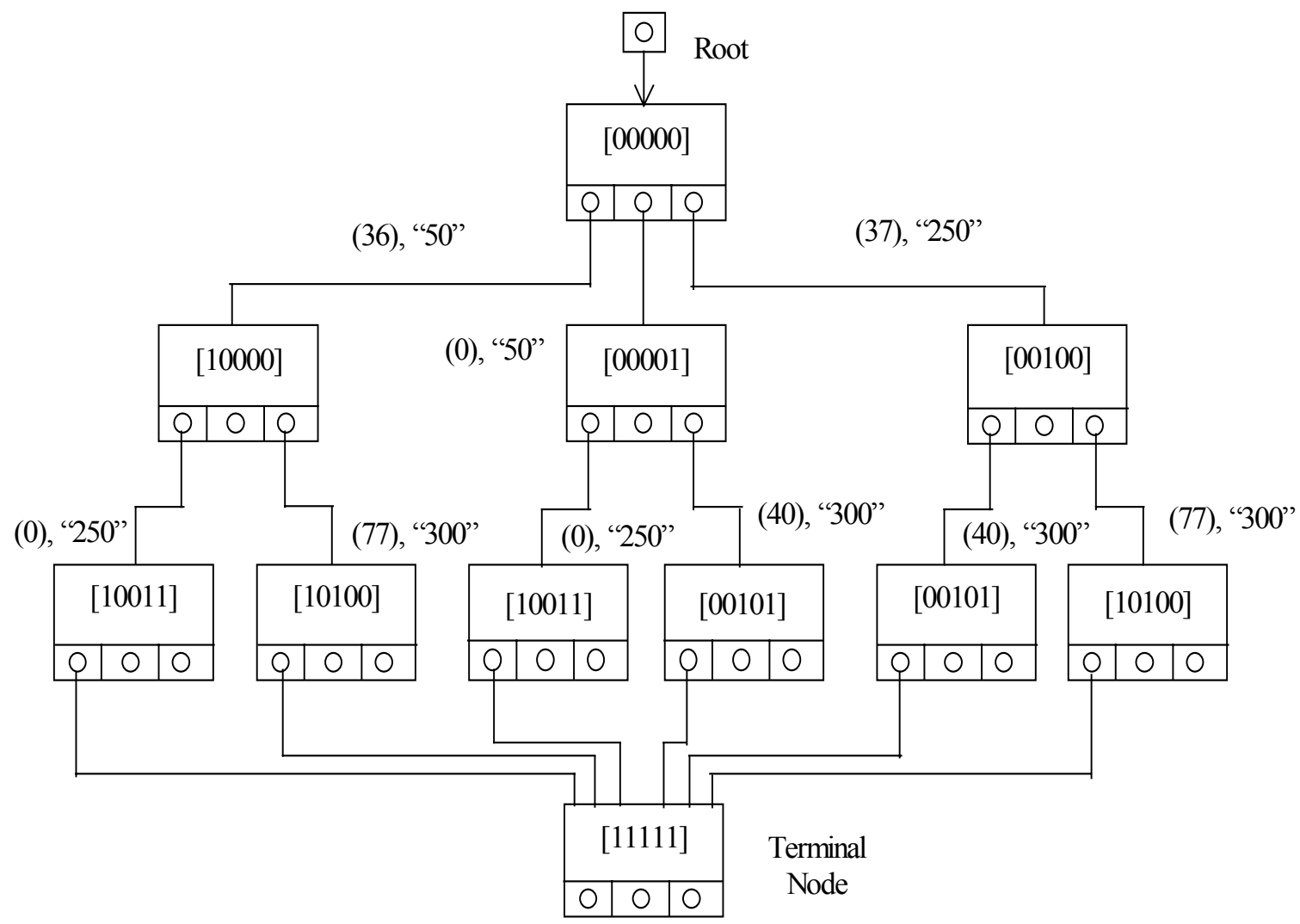

Figure 9. The weight and degree of freedom costs for pincer system

Fig. 9 shows that the third assembly sequence is optimum "0" weight cost and "300" degree of freedom cost. Moreover, the sixth assembly sequence is the least preferable sequence in the feasible assembly sequences. In the optimiza- 
tion approach, both optimization criteria indicated that assembly sequence is optimum.

Therefore, the optimum assembly sequence for pincer system is

$$
\text { [00000],[00001],[10011],[11111]. }
$$

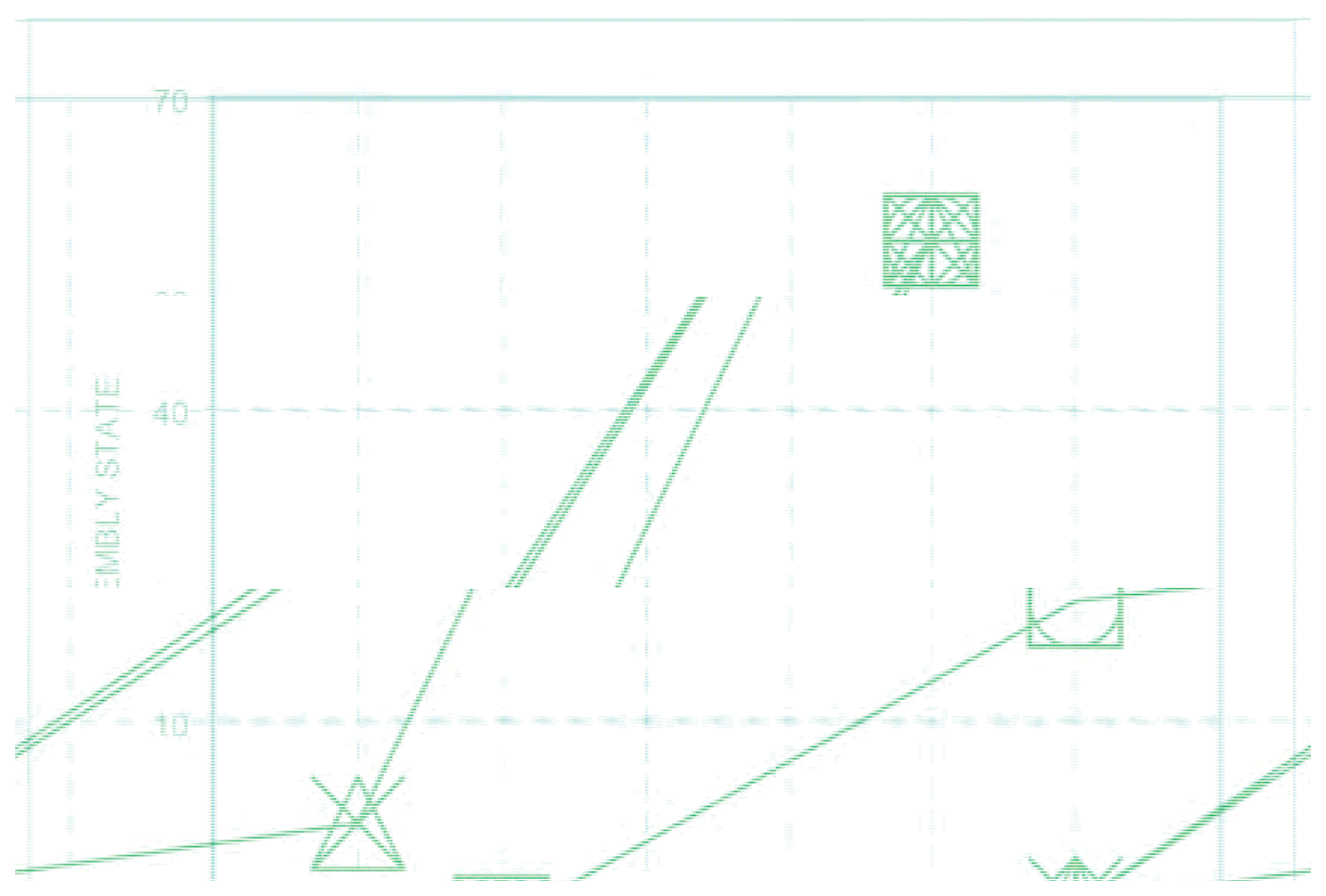

Figure 10(a). The desired feasible assembly sequences for pincer assembly system

Fig. 10(a) (Case 1) shows the desired feasible assembly sequences for pincer assembly system. Fig. 10(b) (Case 2) is also shows these feasible assembly sequences for neural network approach. 


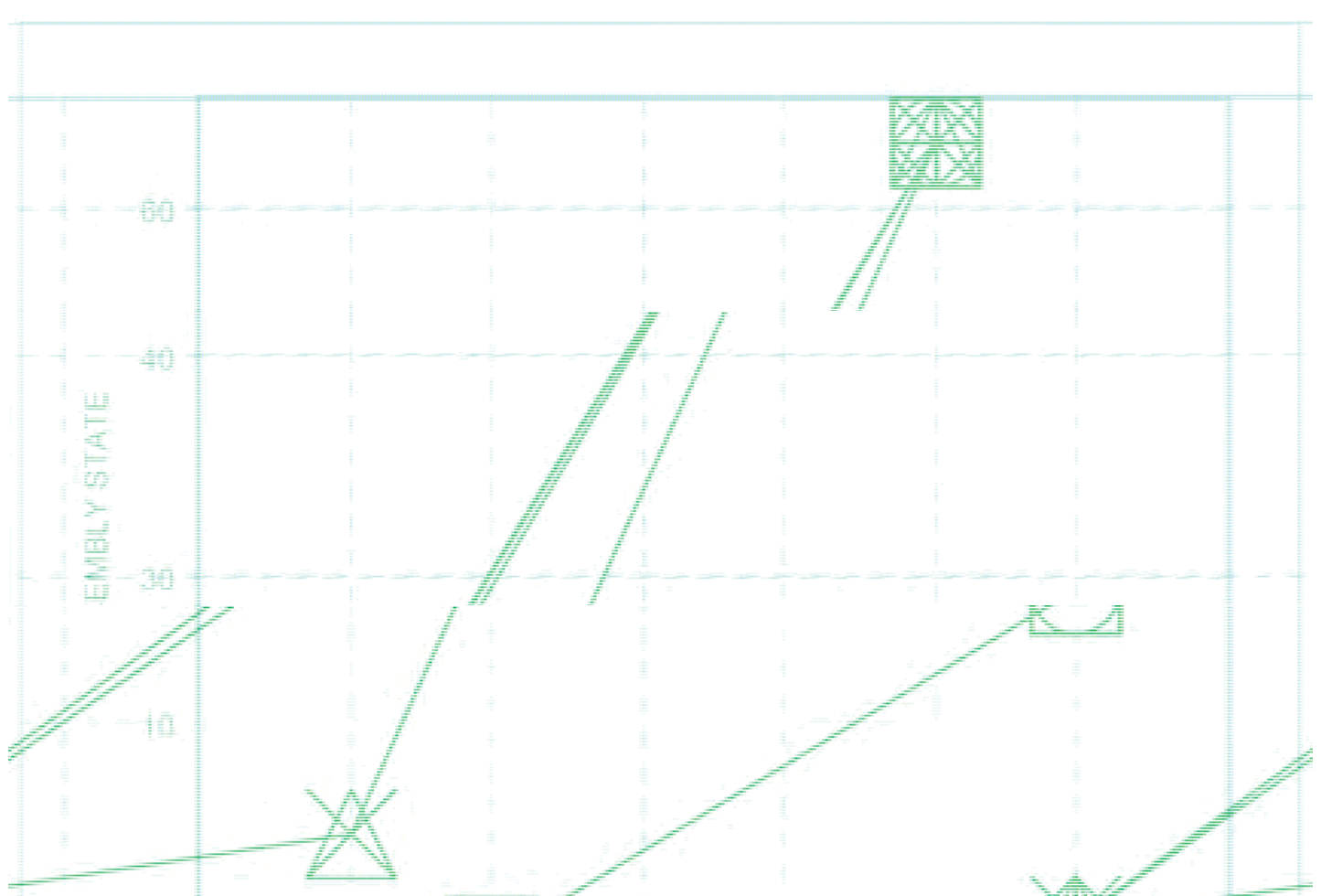

Figure $10(\mathrm{~b})$. The feasible assembly sequences for proposed neural networks approach.

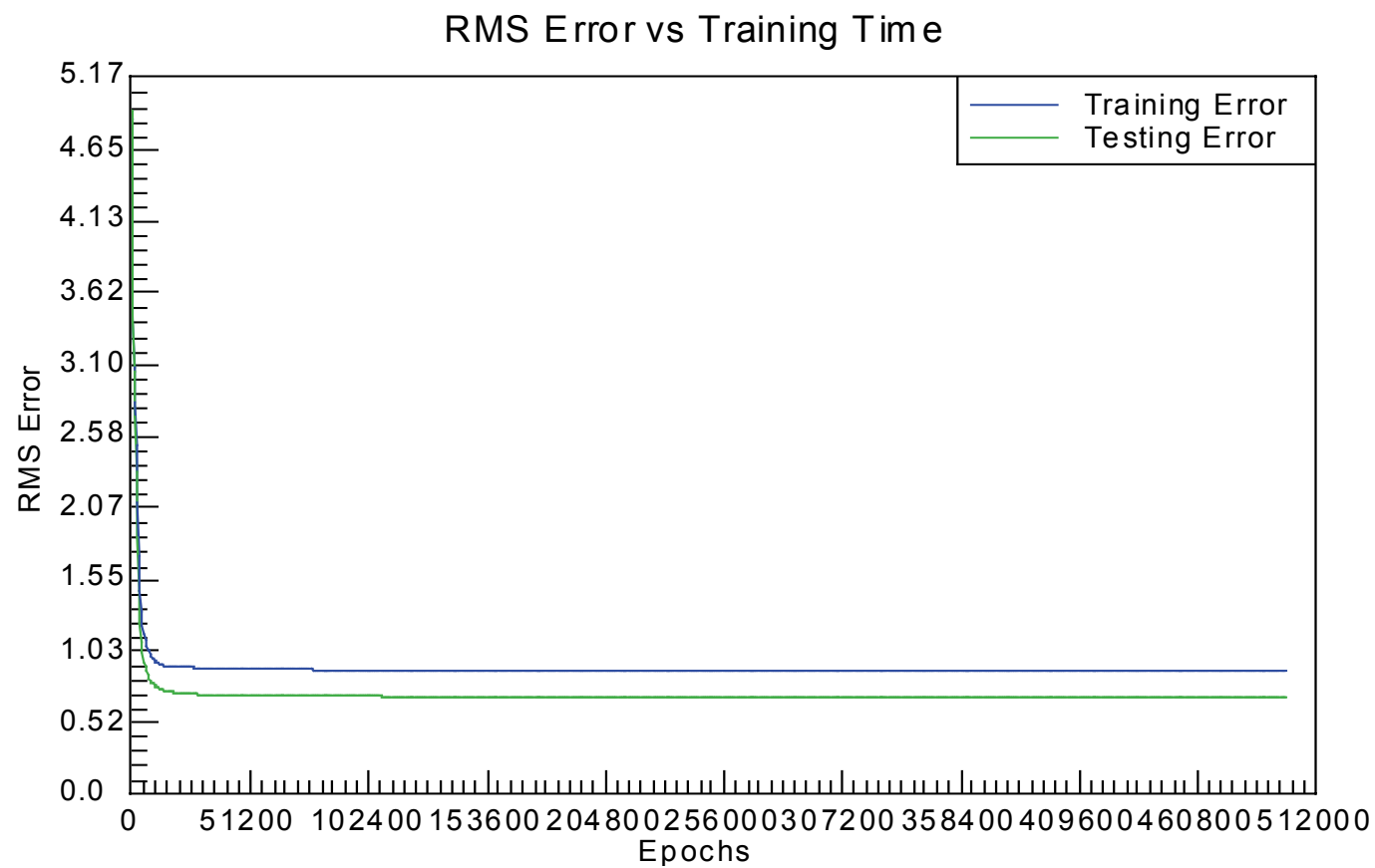

Figure 11. The error convergence graph of the case 2 
The error convergence graph of the case 2 is depicted in Fig. 11 during the training of the network. As can be seen from the figure, the error is suddenly reducing to small values. Small epoch can be employed for case 2 (51200 epoch).

\section{Some Assembly Case Studies}

In this work, some sample assembly systems are examined. Among these examples, four-part hinge system and seven-part coupling system have been investigated. Fig. 12 shows this assembly's exploded views and $A C G$.

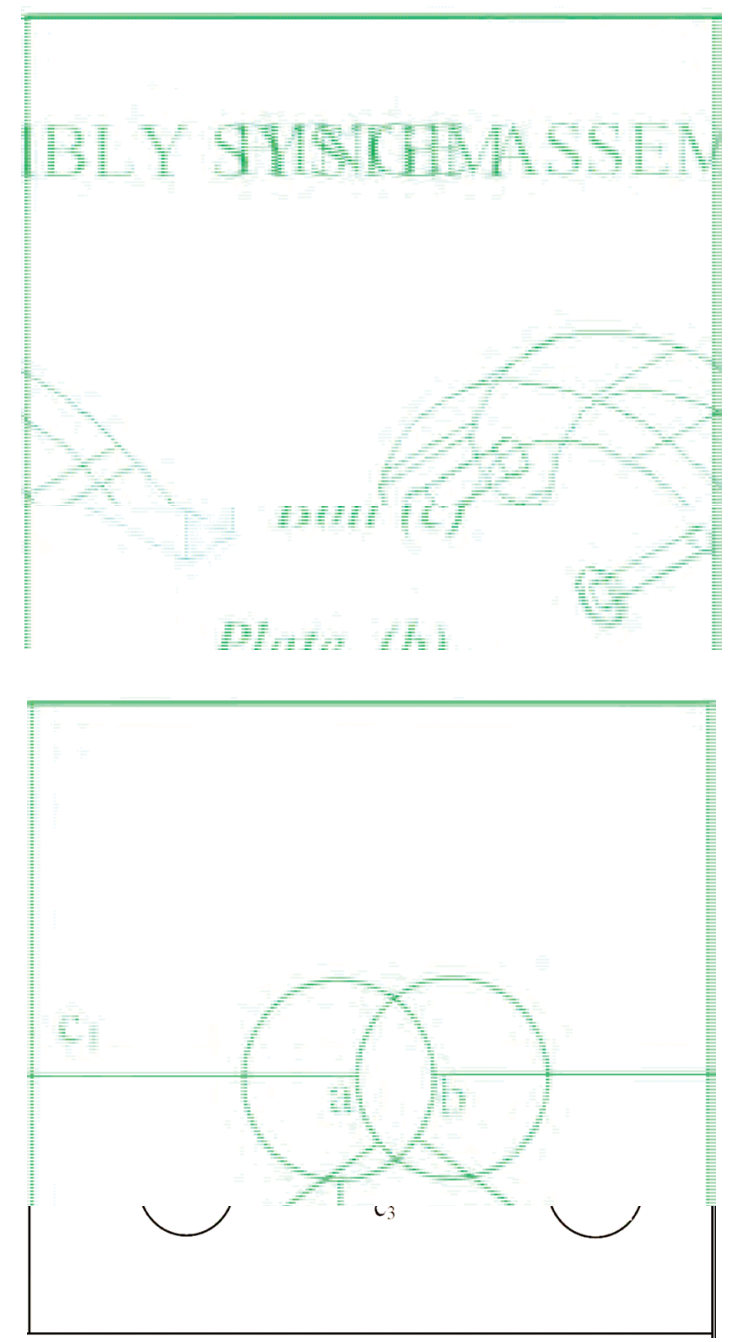

Nodes of $A C G: a$; Handle, $b$; Plate, $c$; Bolt, $d$; Nut 

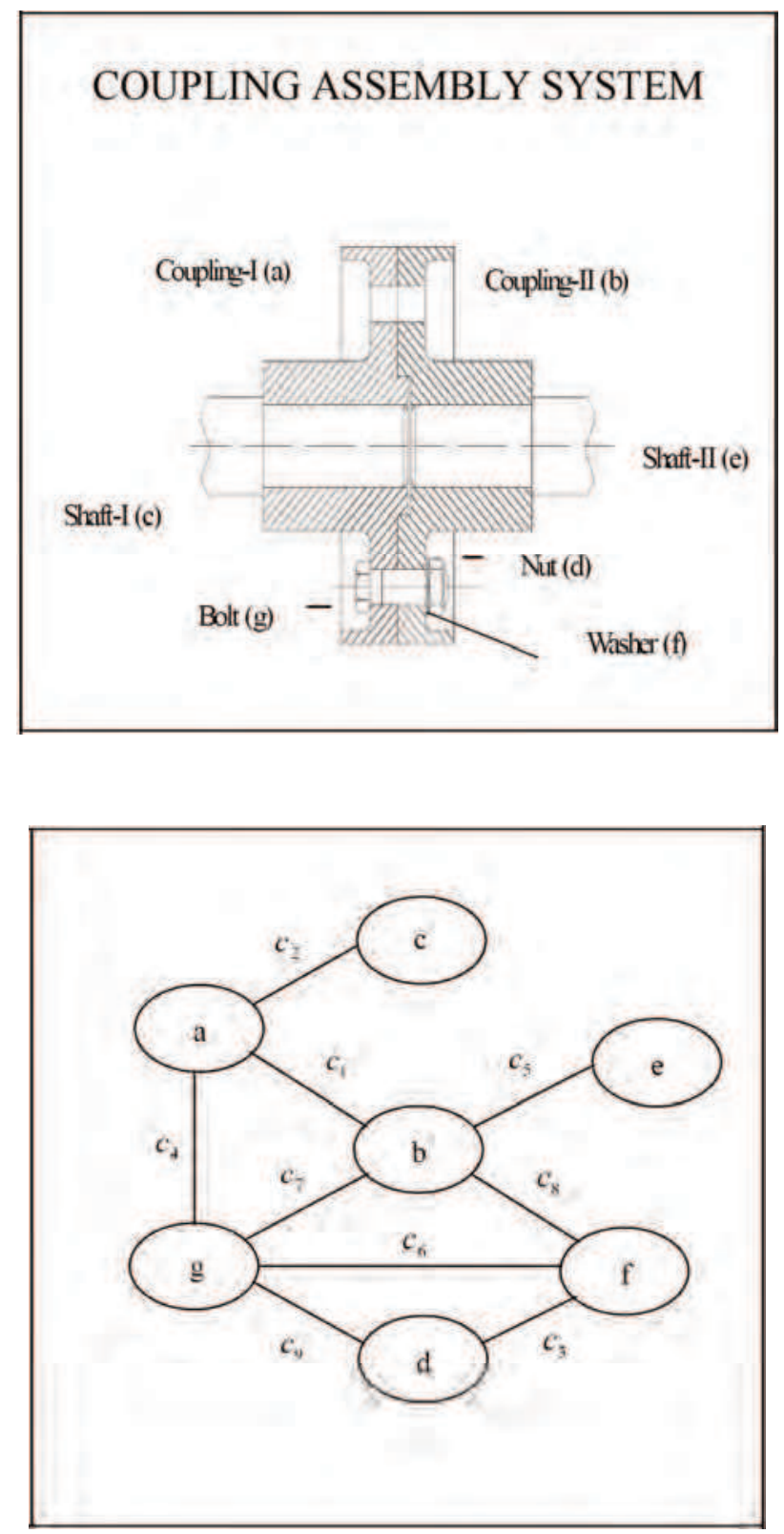

Nodes of $A C G$ : $a$; Coupling-I, $b$; Coupling-II, $c$; Shaft-I, $d$; Nut, $e$; Shaft-II, $f$; Washer, $g$; Bolt

Figure 12. The hinge and coupling systems and their $A C G$ 
The assembly sequences of the hinge system contain four different assembly states. The first one is [00000] and the last is [11111]. Fig. 13 shows feasible assembly sequences for hinge system.

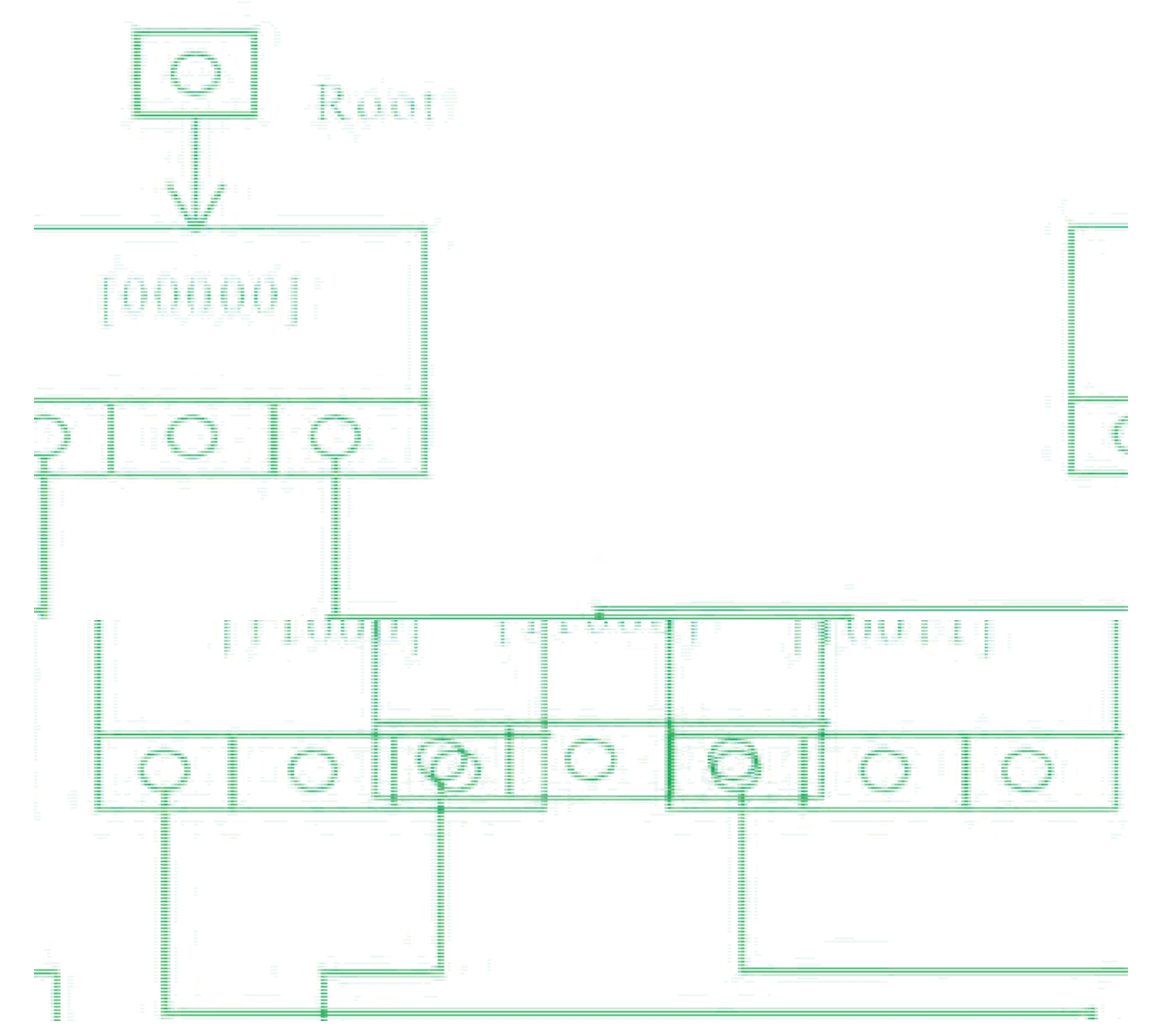

Figure 13. The feasible assembly sequences for hinge system

For instance, in the second assembly sequence, the plate and handle are connected with the connection of $c_{1}$. Then using bolt this subassembly is fixed with the connections of $c_{4}, c_{5}$. And the assembly process is completed with the addition of nut.

In the coupling assembly system, [000000000] and [111111111] are the same as all assembly sequences. Some of the feasible assembly sequences for coupling system are shown in Table 7 . One of the feasible assembly sequences for coupling system is:

$$
\text { [000000000],[010000000],[010010000],[010110000],[111111111] }
$$


FEASIBLE ASSEMBLY SEQUENCES FOR COUPLING SYSTEM

\begin{tabular}{|l|l|l|l|c|l|l|}
\hline 000000000 & 010000000 & 010010000 & \multicolumn{3}{|c|}{011010000} & 111111111 \\
\hline 000000000 & 010000000 & 010010000 & 011010000 & 011010010 & 111010010 & 111111111 \\
\hline 000000000 & 010000000 & 010010000 & 011010000 & 011010010 & 111111111 \\
\hline 000000000 & 010000000 & 010010000 & \multicolumn{3}{|c|}{010110000} & 11111111 \\
\hline 000000000 & 010000000 & 010010000 & 010110000 & 010111000 & 111111111 \\
\hline 000000000 & 010000000 & 010010000 & 010110000 & 010110010 & 111111111 \\
\hline 000000000 & 010000000 & 010010000 & 010110000 & 010110001 & 111111111 \\
\hline 000000000 & 01000000 & 010010000 & 010011000 & 110011000 & 111111111 \\
\hline
\end{tabular}

Table 7. Several assembly sequences of coupling systems

The coupling-I is joined to the shaft-I in the first assembly state; the coupling-II and the shaft-II are added in second assembly state. In the third assembly state, the bolt is used to fix this subassembly, and then the washer and nut is joined to last subassembly.

\section{Conclusions}

In this chapter, an assembly sequence planning system, based on binary vector representations, is explained and some assembly systems are examined. Among these examples, six assembly sequences for four-part pincer system; three assembly sequences for four-part hinge system; three hundred and seventy three assembly sequences for seven-part coupling system have been investigated. NN application was presented for analyzing assembly sequences on assembly system. As can be depicted from the results, the neural predictor would be used as a predictor for possible assembly system applications. The properties and originalities of the developed system;

\section{Easy Representation}

- The representation of assembly models by $A C G$

- The representation of contact and interference relations between parts by interference matrices

- The representation of assembly steps by binary vectors 
Automatic Production

- Contact and interference matrices

- $A C G$

- All binary vectors

- Assembly states

- Geometric feasibility of assembly states

- The automatic determination of assembly states that have the conditions of stability and subassembly

- The automatic determination of feasible assembly sequence plans and optimum assembly one using NN

Practical use

- It is possible to obtain appropriate assembly sequence plans after giving various assembly drawings of a product

- It is understood that the determination of assembly sequence plans that save time and cost after the application of the developed systems to various products

\section{References}

Canbulut, F. \& Sinanoğlu, C. (2004). An Investigation on the Performance of Hydrostatic Pumps Using Artificial Neural Network. JSME International Journal Series C, Vol. 47, 864-872, ISSN: 1344-7653.

De Fazio, TL. \& Whitney, DE. (1987). Simplified Generation of All Mechanical Assembly Sequences. IEEE Transaction on Robotics and Automation, Vol. 3, No. 6, 640-658, ISSN: 1552-3098.

Homem de Mello, LS. \& Arthur, CD. (1990). AND/OR Graph Representation of Assembly Plans. IEEE Transaction On Robotics and Automation; Vol. 6, No. 2, 188-199, ISSN: 1552-3098.

Homem de Mello, LS. \& Lee, S. (1991). Computer Aided Mechanical Assembly Planning, Kluwer Academic Publishers, ISBN: 0792392051, Massachusetts.

Homem de Mello, LS. \& Sanderson, AC. (1991). A Correct and Complete Algorithm for The Generation of Mechanical Assembly Sequences, IEEE Transaction On Robotics and Automation, Vol. 7, No. 2, 228-240, ISSN: 15523098. 
Kandi, S. \& Makino, H. (1996). ASPEN: Computer Aided Assembly Sequence Planning and Evaluation System Based on Predetermined Time Standard, Annals of the CIRP, Vol. 45, No. 1, 35-39, ISSN: 1726-0604.

Pahl, G. \& Beitz, W. (1988). Engineering Design, The Design Council, SpringerVerlag, ISBN: 3-540-50442-7, London.

Sinanoğlu, C., Kurban, AO. \& Yıldırım, Ş. (2004). Analysis of Pressure Variations on Journal Bearing System Using Artificial Neural Network, Industrial Lubrication and Tribology, Vol. 56, No.2, 74-87, ISSN: 0036-8792.

Sinanoğlu, C. \& Börklü, HR. (2004). An Approach To Determine Geometric Feasibility To Assembly States by Intersection Matrices in Assembly Sequence Planning. Journal of Intelligent Manufacturing, Vol. 15, 543-59, ISSN: 0956-5515.

Sinanoğlu, C. \& Börklü, H.R. (2005). An Assembly Sequence Planning System for Mechanical Parts Using Neural Network, Assembly Automation, Vol. 25, No. 1, 38-52, ISSN: 0144-5154.

Singh, N. (1997). Systems Approach to Computer Integrated Design and Manufacturing. John Wiley \& Sons Inc., ISBN: 0-471-58517-3, International Edition. 


\section{Manufacturing}

the Future

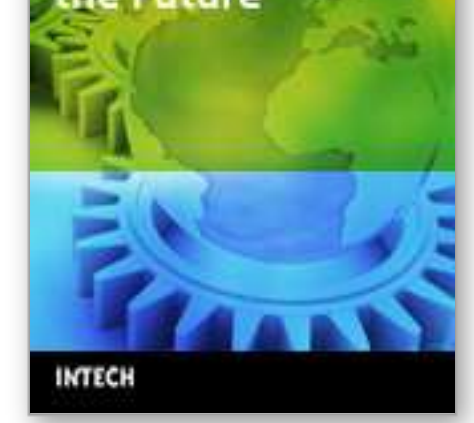

\section{Manufacturing the Future}

Edited by Vedran Kordic, Aleksandar Lazinica and Munir Merdan

ISBN 3-86611-198-3

Hard cover, 908 pages

Publisher Pro Literatur Verlag, Germany / ARS, Austria

Published online 01, July, 2006

Published in print edition July, 2006

The primary goal of this book is to cover the state-of-the-art development and future directions in modern manufacturing systems. This interdisciplinary and comprehensive volume, consisting of 30 chapters, covers a survey of trends in distributed manufacturing, modern manufacturing equipment, product design process, rapid prototyping, quality assurance, from technological and organisational point of view and aspects of supply chain management.

\section{How to reference}

In order to correctly reference this scholarly work, feel free to copy and paste the following:

Cem Sinanoglu and Huseyin Riza Borklu (2006). Assembly Sequence Planning Using Neural Network Approach, Manufacturing the Future, Vedran Kordic, Aleksandar Lazinica and Munir Merdan (Ed.), ISBN: 386611-198-3, InTech, Available from:

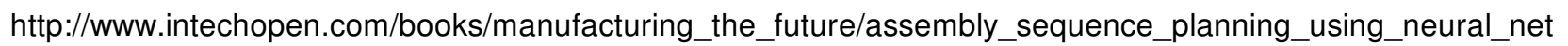
work_approach

\section{INTECH}

open science | open minds

\section{InTech Europe}

University Campus STeP Ri

Slavka Krautzeka 83/A

51000 Rijeka, Croatia

Phone: +385 (51) 770447

Fax: +385 (51) 686166

www.intechopen.com

\section{InTech China}

Unit 405, Office Block, Hotel Equatorial Shanghai No.65, Yan An Road (West), Shanghai, 200040, China 中国上海市延安西路65号上海国际贵都大饭店办公楼405单元 Phone: +86-21-62489820

Fax: $+86-21-62489821$ 
(C) 2006 The Author(s). Licensee IntechOpen. This chapter is distributed under the terms of the Creative Commons Attribution-NonCommercial-ShareAlike-3.0 License, which permits use, distribution and reproduction for non-commercial purposes, provided the original is properly cited and derivative works building on this content are distributed under the same license. 